\title{
ERRATUM
}

\section{DNA sequencing using electrical conductance measurements of a DNA polymerase}

Yu-Shiun Chen, Chia-Hui Lee, Meng-Yen Hung, Hsu-An Pan, Jin-Chern Chiou and G. Steven Huang

Nature Nanotechnology 8, 452-458 (2013); published online 5 May 2013; corrected after print 11 July 2013.

In the version of this Article originally published, the addresses of all affiliations were incorrect; the correct addresses should have read "1Department of Biological Science and Technology, National Chiao Tung University, 1001 University Road, Hsinchu, Taiwan, ROC, ${ }^{2}$ Biomedical Electronics Translational Research Center, National Chiao Tung University, 1001 University Road, Hsinchu, Taiwan, ROC, ${ }^{3}$ Department of Materials Science and Engineering, National Chiao Tung University, 1001 University Road, Hsinchu, Taiwan, ROC, ${ }^{4}$ Institute of Electrical and Control Engineering, National Chiao Tung University, 1001 University Road, Hsinchu, Taiwan, ROC.” In the Acknowledgements, the first sentence should have read "This work was partially supported by the 'Aim for the Top University Plan' of the National Chiao Tung University and the Ministry of Education, Taiwan, ROC." These errors have been corrected in the HTML and PDF versions. 